\title{
Mucormycosis in a 40-year-old woman with diabetic ketoacidosis
}

\author{
Lauren Thomas MD MPH, Sze Yen Tay MBBS BMedSc, Diane Howard MBBS, Henrik Falhammar MD PhD
}

Cite as: CMAJ 2020 April 20;192:E431-3. doi: 10.1503/cmaj.191364

A 40-year-old Indigenous Australian woman was airlifted to hospital from a remote community. She had central chest pain, dyspnea, pedal edema and diabetic ketoacidosis, which she had developed after she stopped taking insulin because of her chest pain. Anxious to remain in her community, she had initially declined an earlier transfer but agreed 3 days later because her diabetic ketoacidosis was deteriorating despite additional insulin. Her medical history included type 1 diabetes, stage 1 chronic kidney disease, hypertension and asthma. She was on a basal-bolus insulin regimen, ramipril, amlodipine and amitriptyline. Her glycosylated hemoglobin at the time of admission was $10.3 \%$.

On examination, the patient was lethargic and tachypnoeic (respiratory rate 23 breaths per minute). She had signs of congestive cardiac failure, including raised jugular venous pressure, bibasal pulmonary crackles and pitting edema to her knees. Her heart sounds were normal.

On investigation, her electrocardiogram showed new T-wave inversion in the inferolateral leads. She had a blood pH of 7.02 (reference range 7.35-7.45) with high anion gap, plasma glucose 38 (reference range $4.0-7.8$ ) $\mathrm{mmol} / \mathrm{L}$, plasma ketones 7.6 (nor$\mathrm{mal}<0.6) \mathrm{mmol} / \mathrm{L}$, high-sensitivity troponin elevation from 199 to 264 (normal < 13) ng/L, hypophosphatemia 0.30 (reference range 0.75-1.50) $\mathrm{mmol} / \mathrm{L}$, hyponatremia 120 (reference range 135145) $\mathrm{mmol} / \mathrm{L}$, serum potassium 4.8 (reference range $3.5-4.5$ ) $\mathrm{mmol} / \mathrm{L}$ and serum creatinine 175 (reference range $60-110) \mu \mathrm{mol} / \mathrm{L}$. She had neutrophilia of 13 (reference range $2.0-7.5$ ) $\times 10^{9} / \mathrm{L}$. Her C-reactive protein was 28.5 (normal < 5.0) $\mathrm{mg} / \mathrm{L}$. HIV serology was negative. A chest radiograph showed peribronchial cuffing with no focal consolidation. A transthoracic echocardiogram revealed moderate left ventricular hypertrophy. We thought that her diabetic ketoacidosis had been precipitated by an acute myocardial infarction. The patient declined to undergo a cardiac angiogram.

Within 24 hours, her diabetic ketoacidosis had resolved with fluid and insulin infusion. On the third day of admission, she reported bloody sputum, sharp right-sided chest pain and painful swallowing.

Chest computed tomography (CT) showed a $2.2 \times 2.0-\mathrm{cm}$ right lower-lobe cavitary mass (Figure 1). Sputum microscopy showed pauci-septated filamentous fungi, which subsequently grew Rhizopus microsporus, a Mucorales species (Figure 2). The minimum inhibitory concentrations for amphotericin $B$ and posaconazole were $0.5 \mathrm{mg} / \mathrm{L}$ and $1 \mathrm{mg} / \mathrm{L}$, respectively.

\section{KEY POINTS}

- Mucormycosis is almost exclusively an opportunistic infection that occurs in the setting of immunosuppression.

- Rapidly progressive or persistent infective symptoms despite broad-spectrum antibiotics in high-risk individuals should prompt investigation for invasive fungal infection such as mucormycosis.

- More than 1 infection may be present in a patient, particularly in those who are immunosuppressed.

Bronchoscopy showed "cheesy" endobronchial material (Figure 3). Culture from bronchoalveolar lavage samples grew $R$. microsporus and methicillin-sensitive Staphylococcus aureus. Gastroscopy showed esophageal candidiasis.

We treated the patient promptly with intravenous liposomal amphotericin B $(5 \mathrm{mg} / \mathrm{kg} / \mathrm{d})$ and flucloxacillin. There were no signs or symptoms of disseminated mucormycosis on her skin or mouth, and nasal endoscopic examination showed no necrotic lesion. She had no history of nasal congestion. Her neurological examination was normal.

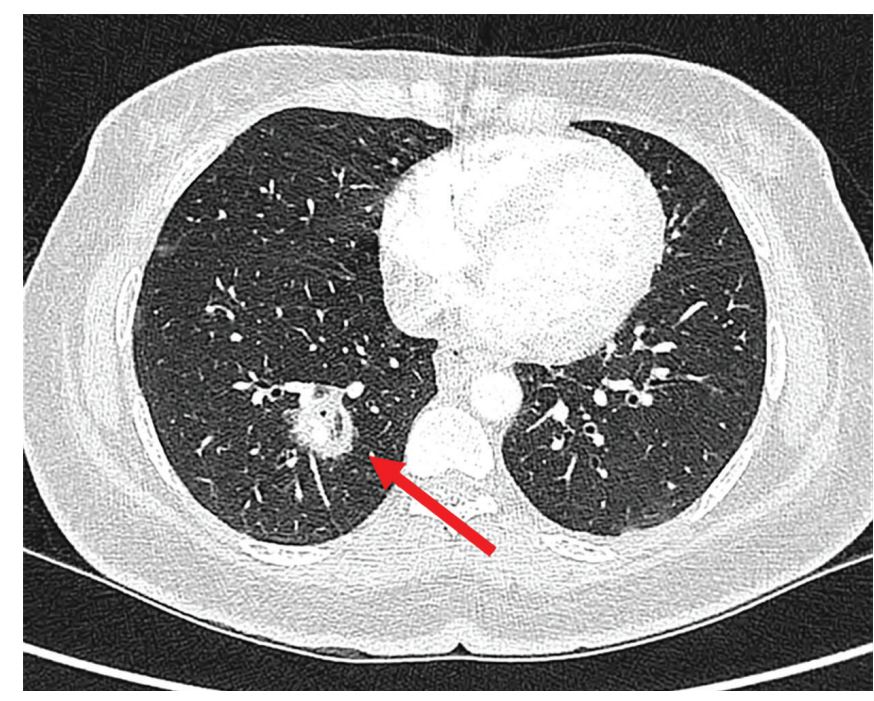

Figure 1: Chest computed tomography scan showing cavitary lesion (arrow) in a 40-year-old woman who subsequently received a diagnosis of mucormycosis. 


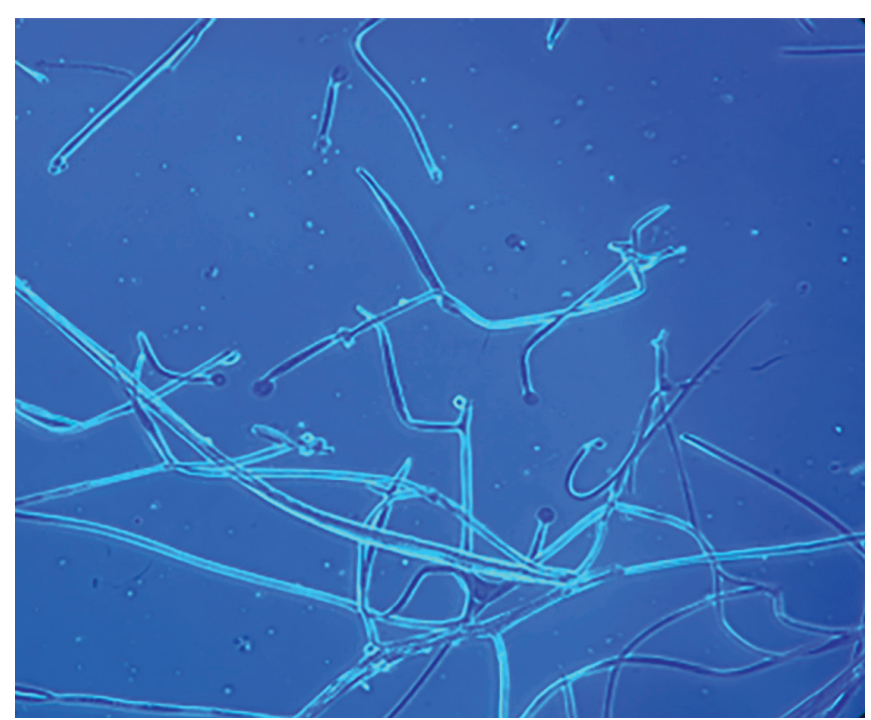

Figure 2: Microscopy of sputum sample containing aseptate hyphae suggestive of Rhizopus species.

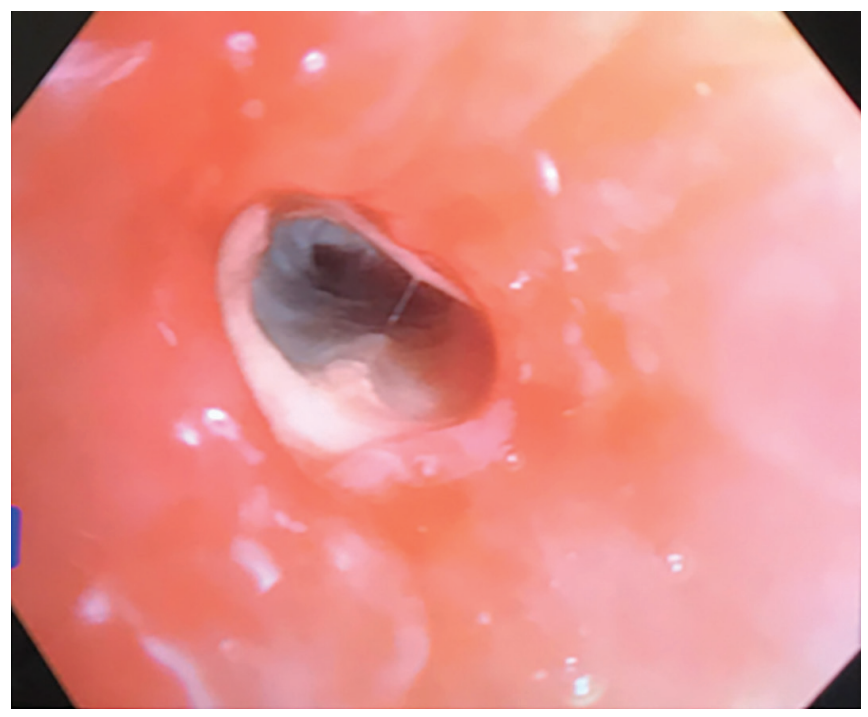

Figure 3: Photo from bronchoscopy showing endobronchial "cheesy" material.

Despite amphotericin and flucloxacillin, the patient developed a fever as a result of a new, left lower-lobe, hospital-acquired pneumonia, which resolved after intravenous piperacillin-tazobactam was added. Repeat sputum culture had no growth.

After 2 weeks of intravenous liposomal amphotericin B, the patient returned home on a 6-week course of posaconazole (300 mg daily dosing after an initial loading dose). Post-treatment $\mathrm{CT}$ at 3 months showed resolution of her pulmonary infection.

\section{Discussion}

Mucormycosis (zygomycosis) is an angioinvasive mould infection caused by a group of ubiquitous fungi (Mucorales). Mucormycosis is an opportunistic infection that occurs almost exclusively in people who are immunosuppressed. Common risk factors include poorly controlled diabetes, malignancy, prolonged neutropenia, high-dose steroid use, transplantation, iron overload and malnourished states as seen in chronic renal failure, HIV infection and low-birth-weight infants. ${ }^{1}$ Mucormycosis may occur in healthy individuals by direct inoculation through broken skin, as occurs in intravenous drug users, injury and burns. ${ }^{1}$ Nosocomial outbreaks may occur with transmission through contaminated bandages, tongue depressors and intravenous catheters. ${ }^{1}$

\section{Importance of early diagnosis}

Mucormycosis is often a postmortem diagnosis. It is aggressive and difficult to diagnose. Early diagnosis and treatment are essential, as a delay of even 6 days is associated with a doubling of 30 -day mortality, from $35 \%$ to $66 \%{ }^{2}$

\section{Changing epidemiology}

The reported incidence varies from 0.005 to 1.7 per million population, depending on the geographical region and study centre. ${ }^{1}$ But this may be an underestimate, as mucormycosis was diagnosed in as many as 5 per 10000 postmortem cases in an autopsy audit. ${ }^{3}$

Diabetes is the most common risk factor, but this is changing - especially in developed countries, where malignant disease and related immunosuppression have replaced diabetes as the predominant risk factors. ${ }^{4,5}$ However, poorly controlled diabetes still contributes to $75 \%$ of mucormycosis cases in low-income countries. ${ }^{4,5}$ The global mucormycosis case-fatality rate is $46 \%,{ }^{4}$ but is lower in patients with diabetes compared with those with hematological malignancy, in whom dissemination of mucormycosis is more common.

\section{Diabetic ketoacidosis and mucormycosis}

Mucorales require iron for growth, must evade host phagocytic defence mechanisms and access the vasculature to disseminate. Phagocytic activities become dysfunctional in the hyperglycemic state of diabetic ketoacidosis. In addition, free serum iron also increases owing to impaired transferrin binding in hyperglycemic acidosis. ${ }^{6}$

Additionally, Mucorales have a ketone reductase system that allows them to thrive in hyperglycemic and acidotic conditions, ${ }^{7}$ perhaps explaining the disproportionally higher incidence of mucormycosis in patients with diabetic ketoacidosis.

\section{Clinical manifestations}

Mucorales may infect any organ. Rhino-orbital-cerebral infection is most common in diabetes, whereas pulmonary and disseminated infections are more common in patients with malignant disease and transplantation recipients. ${ }^{4}$ Initial symptoms may be nonspecific. In high-risk individuals, invasive fungal infection should be suspected if there is hemoptysis, pleuritic chest pain, unilateral facial pain or swelling, orbital swelling, or proptosis. Other presentations include nonresolving infective symptoms despite broad-spectrum antibiotics or voriconazole prophylaxis and the presence of necrotic tissue (e.g., palatine or nasal eschars, cutaneous necrosis). Tissue necrosis, often a late sign, is a hallmark of mucormycosis, resulting from angioinvasion and vascular thrombosis. 


\section{Radiological investigation}

In pulmonary disease, high-resolution CT is the preferred test with findings that include diffuse opacities, cavitary lesions, the "halo" sign and large pulmonary wedge infarcts. ${ }^{8}$ The presence of more than 10 pulmonary nodules, pleural effusion and "reverse halo" signs points to mucormycosis rather than the more common invasive fungal infection of aspergillosis. ${ }^{1,8}$ Radiological changes in early rhino-orbital-cerebral disease may be nonspecific.

\section{Diagnosis}

Histopathologic examination is essential. Tissue invasion, in particular angioinvasion, is highly suggestive of active disease rather than fungal colonization or culture contamination. Histology of fresh, frozen or formalin-fixed samples may offer a faster diagnosis. Culture is important for species identification and antifungal susceptibility testing. There is no recommended biomarker, although serum disaccharide testing appears promising. ${ }^{1}$ Fungal cell-wall biomarkers such as galactomannan and 1,3- $\beta$-D-glucan are absent, or present in low quantities in Mucorales species, respectively; a positive test suggests non-Mucorales fungi.

\section{Management}

Empirical antifungal treatment should be commenced immediately and surgical opinion sought.

Intravenous amphotericin is the antifungal therapy of choice., ${ }^{1,9}$ Posaconazole and isavuconazole are potential alternatives to amphotericin, especially in the maintenance phase. Posaconazole is less toxic and exists in a tablet form, and hence may be used in combination with amphotericin to allow for early hospital discharge. Although the new posaconazole tablet formulation has better bioavailability than the previous oral suspension, regular posaconazole monitoring is still strongly recommended in a new global consensus guideline, to reduce risk of treatment failure. ${ }^{1}$ The optimal therapeutic drug level is yet to be defined; posaconazole trough concentration of $1 \mu \mathrm{g} / \mathrm{mL}$ or higher is currently recommended. ${ }^{1}$

Tissue necrosis can prevent tissue penetration of antifungal agents..$^{10}$ Hence, surgical débridement of necrotic tissue is essential. A recent meta-analysis showed that the combination of surgical débridement and antifungal therapy was associated with significantly lower 90-day mortality compared with using antifungal therapy alone. ${ }^{9}$

Antifungal treatment duration is guided by resolution of clinical and radiological features. Reversal of underlying risk factors may improve survival. Multidisciplinary and expert involvement are strongly recommended. ${ }^{1}$

\section{References}

1. Cornely OA, Alastruey-Izquierdo A, Arenz D, et al.; Mucormycosis ECMM MSG Global Guideline Writing Group. Global guideline for the diagnosis and management of mucormycosis: an initiative of the European Confederation of Medical Mycology in cooperation with the Mycoses Study Group Education and Research Consortium. Lancet Infect Dis 2019;19:e405-21.

2. Chamilos G, Lewis RE, Kontoyiannis DP. Delaying amphotericin B-based frontline therapy significantly increases mortality among patients with hematologic malignancy who have zygomycosis. Clin Infect Dis 2008;47:503-9.

3. Hotchi M, Okada M, Nasu T. Present state of fungal infections in autopsy cases in Japan. A statistical survey of all autopsy cases during the ten-year period from 1966 to 1975. Am J Clin Pathol 1980;74:410-6. doi: 10.1093/ajcp/74.4.410.

4. Jeong W, Keighley C, Wolfe R, et al. The epidemiology and clinical manifestations of mucormycosis: a systematic review and meta-analysis of case reports. Clin Microbiol Infect 2019;25:26-34.

5. Prakash H, Chakrabarti A. Global epidemiology of mucormycosis. J Fungi (Basel) 2019;5:pii: E26.

6. Ibrahim AS. Host-iron assimilation: pathogenesis and novel therapies of mucormycosis. Mycoses 2014;57(Suppl 3):13-7.

7. Ribes JA, Vanover-Sams CL, Baker DJ. Zygomycetes in human disease. Clin Microbiol Rev 2000;13:236-301.

8. Georgiadou SP, Sipsas NV, Marom EM, et al. The diagnostic value of halo and reversed halo signs for invasive mold infections in compromised hosts. Clin Infect Dis 2011;52:1144-55.

9. Jeong W, Keighley C, Wolfe R, et al. Contemporary management and clinical outcomes of mucormycosis: a systematic review and meta-analysis of case reports. Int J Antimicrob Agents 2019;53:589-97.

10. Spellberg B, Ibrahim AS. Recent advances in the treatment of mucormycosis. Curr Infect Dis Rep 2010;12:423-9.

\section{Competing interests: None declared.}

This article has been peer reviewed.

The authors have obtained patient consent.

Affiliations: Departments of General Medicine (Thomas) and Infectious Diseases (Tay), Endocrinology (Howard), Royal Darwin Hospital, Darwin, Australia; Departments of Molecular Medicine and Surgery, and Endocrinology, Metabolism and Diabetes (Falhammar), Karolinska University Hospital, Stockholm, Sweden

Contributors: All of the authors contributed to the conception and design of the work, and the acquisition, analysis, and interpretation of data. All of the authors drafted the manuscript, revised it critically for important intellectual content, gave final approval of the version to be published and agreed to be accountable for all aspects of the work.

Acknowledgement: The authors thank the consulting infectious disease team, especially Dr. Jane Davies for her clinical advice and follow-up of the patient.

Correspondence to: Sze Yen Tay, szeyentay@gmail.com 